\title{
Automated Identification of Photoreceptor Cones Using Multi-scale Modelling and Normalized Cross-Correlation
}

\author{
Alan Turpin ${ }^{1}$, Philip Morrow ${ }^{1}$, Bryan Scotney ${ }^{1}$, Roger Anderson ${ }^{2}$, \\ and Clive Wolsley ${ }^{2}$ \\ ${ }^{1}$ School of Computing and Information Engineering, Faculty of Computing and Engineering \\ ${ }^{2}$ School of Biomedical Sciences, Faculty of Life and Health Sciences, \\ University of Ulster, Northern Ireland \\ \{turpin-a1, wolsley-c\}@email.ulster.ac.uk, \\ \{pj.morrow, bw.scotney, rs.anderson\}@ulster.ac.uk
}

\begin{abstract}
Analysis of the retinal photoreceptor mosaic can provide vital information in the assessment of retinal disease. However, visual analysis of photoreceptor cones can be both difficult and time consuming. The use of image processing techniques to automatically count and analyse these photoreceptor cones would be beneficial. This paper proposes the use of multiscale modelling and normalized cross-correlation to identify retinal cones in image data obtained from a modified commercially available confocal scanning laser ophthalmoscope (CSLO). The paper also illustrates a process of synthetic data generation to create images similar to those obtained from the CSLO. Comparisons between synthetic and manually labelled images and the automated algorithm are also presented.
\end{abstract}

Keywords: Modelling, Cross Correlation, Multi-Scale, Retinal Cones.

\section{Introduction}

A number of diseases can affect specific components of the eye, including retinitis pigmentosa, cone/cone-rod dystrophy, glaucoma, age-related macular degeneration and many more. Some of these can be visually detected and early treatment, where available, is applied. However those that affect the retina at the back of the eye, such as cone/cone-rod dystrophy and age-related macular degeneration are significantly harder to detect as it is very difficult to obtain images of the photoreceptor mosaic in vivo.

Previously, to view the retinal photoreceptor mosaic a post-mortem donor eye had to be placed under a microscope and cones were then counted. However, with recent advances in technology it is now possible to view the photoreceptor cones through the use of a modified confocal scanning laser ophthalmoscope (CSLO) or adaptive optics (AO) [1] in conjunction with a CSLO. The analysis of such images though can prove time consuming with regard to counting and calculating the density of the retinal cones. 
In previous work in this area $\mathrm{Li}$ and Roorda [2-5] have used $\mathrm{AO}$ in conjunction with a CSLO to capture images of the retinal photoreceptor. The images obtained using this technique clearly shows a hexagonal layout of the photoreceptor cones, however the method does not capture images of the rods as they are significantly smaller than the photoreceptor cones. The images that are produced from their approach clearly illustrate that cone brightness can vary across the entire retina. Images that are obtained from a modified CSLO are substantially different from those captured using Li and Roorda's method in both magnification and pixel resolution. Li and Roorda reported a 93-97\% accuracy of correct photoreceptor cone identification on AO images.

Algorithms created for automated identification of photoreceptor cones on $\mathrm{AO}$ or post-mortem images may not perform as accurately when applied to images captured from a modified CSLO. Wojtas et al [6]achieved an accuracy of $97 \%$ on images obtained from a post-mortem donor eye (where the definition of accuracy is the percentage of the total number of cones correctly identified by the algorithm). They applied background removal and local maxima detection to identify the bright points in each image. The use of directional light was applied at $137^{\circ}$ which in turn was then used to approximate the distance to the cone centre from the local maxima co-ordinate.

Curcio et al [7] studied the topography of the photoreceptor mosaic in post-mortem donor eyes. They found that the density of the human photoreceptor mosaic reduced when moving away from the fovea. Curcio reported a peak foveal density of 199,000 cones $/ \mathrm{mm}^{2}$.

Nicholson and Glaeser [8] have illustrated an approach of normalized crosscorrelation to detect particles in cryo-electron microscopy images. Although this technique is applied to a different type of image, it may be possible to adapt this approach to retinal photoreceptor images. This process has the potential to be used if a model or series of models were created to mimic the shape and size of the photoreceptor cones.

The remained of this paper is organised as follows. Section 2 provides details of the technologies and methodologies used in this research. Section 3 illustrates the design implementation of our algorithms. Section 4 describes a process of synthetic data generation. Section 5 illustrates experimental results against synthetic and real image data and finally Section 6 provides conclusions and outlines further work to be carried out.

\section{Technologies and Methodology}

\subsection{Technologies Available}

The two main technologies that can capture images of the photoreceptor mosaic in vivo are adaptive optics (AO) and the use of a modified CSLO. AO was originally designed to correct for atmospheric distortions in telescope images and has subsequently been developed to correct for ocular distortions [9]. Such a system is large and requires a unique setup for each individual subject that is being imaged. This makes the system expensive and a commercial model has yet to be developed. However, the use of a CSLO such as the Heidelberg Retina Tomorgraph (HRT) [10] is widely available and relatively inexpensive. 
The standard HRT uses a helium neon diode laser with a wavelength of $670 \mathrm{~nm}$. It has a depth resolution of 20-80um and a scanning window of between $10^{\circ}$ and $20^{\circ}$ field of view (FoV) with a resolution of 256x256 pixels. With these parameters the standard HRT cannot discriminate between the retinal layers and is mostly used to evaluate the optic nerve head and nerve fibre layer. Fig. 1 shows a standard HRT image at $10^{\circ} \times 10^{\circ} \mathrm{FoV}$. Using the current $10^{\circ} \times 10^{\circ} \mathrm{FoV}$ scan window setting, the pixel size corresponds to around $10 \mathrm{um}$. However, with significant modification of the HRT involving a reduction in the FoV to a $1^{\circ} \times 1^{\circ}$ scan area, minimizing the scan depth to cover $0.5 \mathrm{~mm}$ and accurate fine focusing it is possible to capture enface images of the cone photoreceptors. Using these settings the pixel size is reduced to close to $1-2 \mu \mathrm{m}$ and successive scan planes are $0.0156 \mathrm{~mm}$ apart. Fig. 2 shows an image captured with the modified HRT at $1^{\circ} \times 1^{\circ} \mathrm{FoV}$. In this image the retinal cones are clearly visible as small, bright circular regions. We can also see retinal blood vessels which appear in the image as the dark region passing from top right to bottom left. The HRT used in this research has been modified with these setting and is located in the Vision Sciences Research labratory at the University of Ulster.

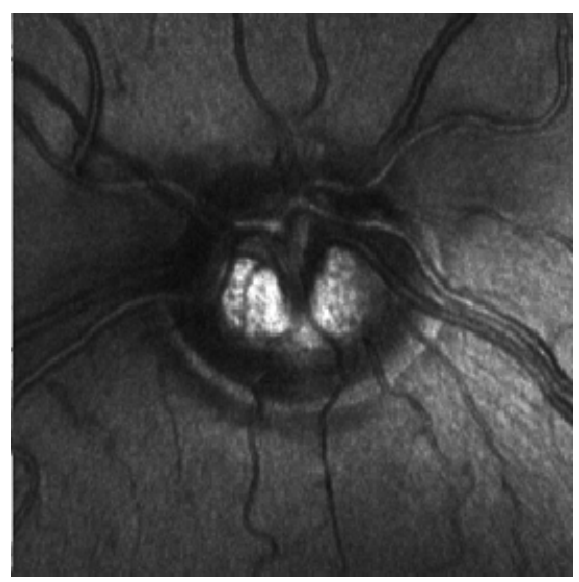

Fig. 1. A standard HRT image captured at $10^{\circ} \times 10^{\circ} \mathrm{FoV}$

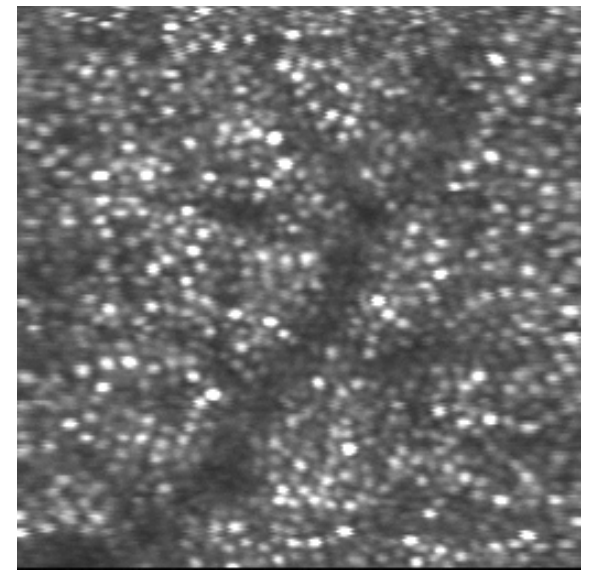

Fig. 2. An example image of retinal cones captured from a modified HRT at $1^{\circ} \times 1^{\circ} \mathrm{FoV}$

\subsection{Methodology}

The overall aim of this research is to automatically analyze and detect photoreceptor cones in an image obtained from a modified CSLO. We aim to achieve this by initially modeling the size and shape of the retinal cones using a Gaussian based model. Normalized cross-correlation is then used to apply the Gaussian model to the retinal images. The output of this approach should highlight all the regions of the image that are similar to the shape of the Gaussian. We then apply local maxima detection to identify the highest points of similarity found in the normalized crosscorrelation results. To validate our results across a large number of images we have 
also generated synthetic data through the use of Gaussian modelling and filtering to replicate the density, size and shape of the retinal cones. A small number of real images have also been manually labeled to act as ground truth data for evaluation purposes. Other approaches such as simple thresholding or applying local maxima detection were implemented however these produced poor results due to the low pixel magnification. We therefore decided to use an approach of Gaussian modelling and normalized cross-correlation to identify similarities in the intensity profiles of the images.

\section{Implementation}

\subsection{Cone Modelling}

The first step in our process is to generate a series of multi-scale Gaussian models that simulate the average shape, size and height of the retinal cones found in the real image data. After extensive analysis of the images we found that the majority of photoreceptor cones can be classified into one of three different types, small and bright, medium size and brightness or large and dull. Fig. 3 shows an example for each of these groups and Fig. 4 illustrates these cones displayed as a 3D surface.

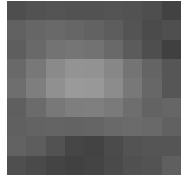

(a)

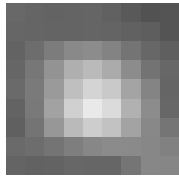

(b)

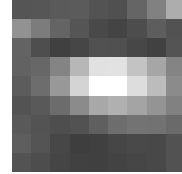

(c)

Fig. 3. Three different types of retinal cones, (a) dull and large, (b) medium size and brightness, (c) bright and small

We can generate a family of Gaussian models to approximate these cone shapes and sizes. A 2D Gaussian model can be defined using equation 1.

$$
G(x, y)=e^{-\frac{x^{2}+y^{2}}{2 \sigma^{2}}} .
$$

where $x$ and $y$ are the size of the model and $\sigma$ controls the spread of the model. The 'sclaing' value that would usually be found in this standard Gaussian formula has been omitted as we wish the values in our models to be always between 0 and 1 . The spread determines if the model with be bright with a sharp decrease or dull and flat. The value of $\sigma$ is specified based on a calculation of the half-height width of the model and is illustrated in equation 2. 


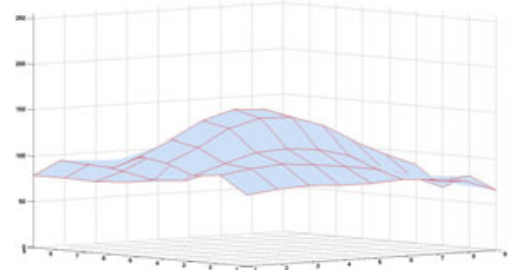

(a)

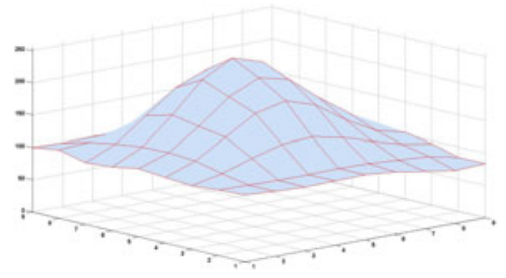

(b)

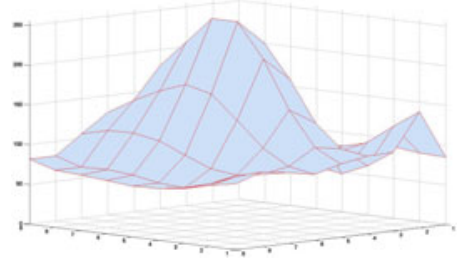

(c)

Fig. 4. The example retinal cones from Fig. 3 displayed as 3D surfaces

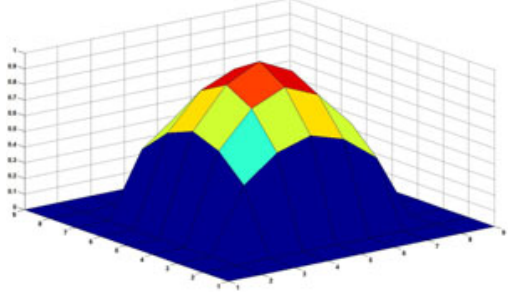

(a)

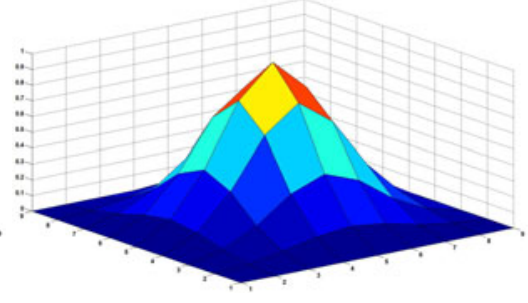

(b)

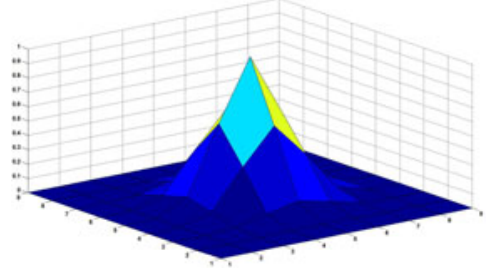

(c)

Fig. 5. Gaussian models for each cone type, (a) dull and large, (b) medium size and brightness, (c) bright and small 


$$
\sigma=\left(-\frac{(m / \gamma)^{2}}{2 \ln (0.5)}\right)^{0.5}
$$

This formula determines the half-height width of the model given a mask size $m$ and a spread $\gamma$. After experimenting with a variety of different sizes and spreads a series of Gaussian models were created, and these are illustrated in Fig. 5.

\subsection{Normalized Cross-Correlation}

Normalized cross-correlation compares a model (illustrated above) to each underlying neighbourhood of an image and returns a value between -1 and 1 at each point in the image. This value illustrates how similar the underlying neighbourhood of the image is when applied with a model. The more similar the underlying neighbourhood is to the model the closer the result will be 1 . If the underlying neighbourhood is inverted with respect to the model, the results will be closer to -1 . The standard normalized cross-correlation equation is illustrated in equation 3.

$$
\operatorname{NCC}(u, v)=\frac{\sum_{x, y}\left(f(x, y)-\bar{f}_{u, v}\right)(t(x-u, y-v)-\bar{t})}{\left\{\sum_{x, y}\left(f(x, y)-\bar{f}_{u, v}\right)^{2} \sum_{x, y}(t(x-u, y-v)-\bar{t})^{2}\right\}^{0.5}} .
$$

where $f$ is the image, $t$ is the mask, $\bar{t}$ is the mean of the mask and $\bar{f}_{u, v}$ is the mean of the region under the mask. As our approach uses multi-scale models we apply each model in turn. This produces three sets of normalized cross-correlation results from which we identify pixels with the highest similarity in each set of results.

\subsection{Centre Identification}

To identify the potential photoreceptor cone centres, we analyze the three normalized cross-correlation results. A local maxima detection algorithm is applied to the normalized cross-correlation results. This identifies all the local maximum pixel values in a neighbourhood of a specified size. The size of this neighbourhood is set to the size of the current Gaussian model. As the local maxima algorithm is applied to the three cross-correlation results, we are presented with three lists of co-ordinates. These three lists are then combined by checking the Euclidian distance of each coordinate. If two or more co-ordinates are within a Euclidian distance of 2 then the average position of these cones is taken. Also, the co-ordinates that are within the Euclidian distance are also marked as used so that they cannot be counted by any further iterations of the algorithm.

\subsection{Post-processing}

After the three lists of co-ordinates have been combined there is stil the possibility that some cone positions have been falsely identified and we must then analyse the coordinate set to remove those co-ordinates which do not correspond to real cone centres. 
To achieve this we firstly average the three normalized cross-correlation results. From this we analyze the co-ordinates in the averaged cross-correlation results, if this coordinate is below a defined threshold the co-ordinate is then removed from the list. This threshold has been chosen emperically and is currently set to 0.3 .

\section{Synthetic Data Generation}

The manually labeling of real image data is both difficult and time consuming, therefore a process of synthetic data generation could be very beneficial. This process is applied as an extension of the modelling process. After analysis of the real image data it was found that most images at $1^{\circ} \times 1^{\circ}$ FoV contain between $600-1000$ photoreceptor cones varying in size, shape and height. Also there is a degree of noise in the real image data caused during image capture. In our approach to generating synthetic data we randomly place Gaussian models on a 256x256 pixel image ensuring that no two are overlapping. The size and spread of the models are randomly selected at 5,7 or 9 and 4, 6 or 8 respectively. These random selections are of equal probability and inevitably there are a large number of different cones across the image with varying densities.

The background in real image data also varies. To generate a synthetic background, a $5 \times 5$ average filter was passed across a real image a large number of times (typically $100)$ in order to produce a smoothed image with varying background. The cones are then randomly placed onto this synthetic background. Fig. 6 illustrates 3 images with cone densities of 600,800 and 1000 .
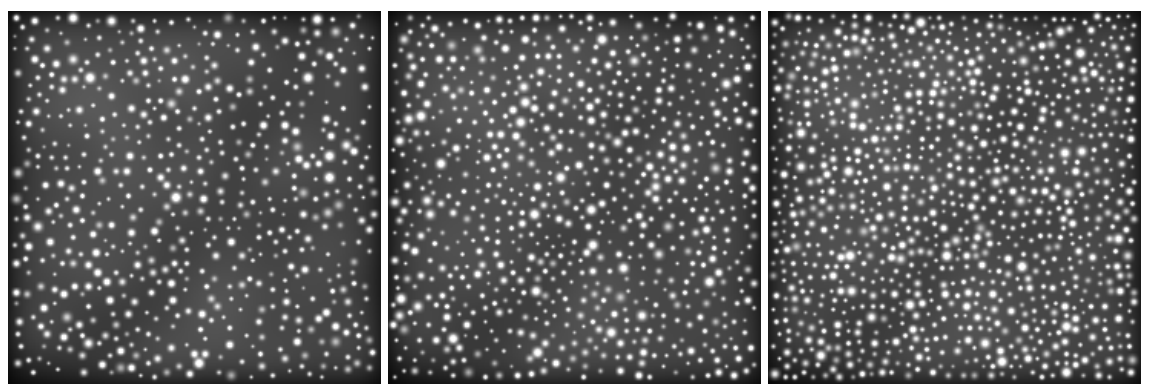

Fig. 6. Three synthetic images with varying densities of 600,800 and 1000 simulated retinal cones

Using this process of generating ideal images a degree of noise can be applied to simulate real image data. For this we choose two different approaches; the first is to apply a $3 \times 3$ Gaussian smoothing algorithm to $70 \%$ of the image. When capturing the images, there can be significant eye movement in any direction. To simulate this we implement a process that applies a $3 \times 3$ pixel mask to the entire image. This mask will shift the centre pixel either up, down, left, right or not at all. Each pixel in the image has a $20 \%$ chance it will be shifted in a direction or not at all. Fig. 7 shows an example of each of these noise filters. 


\section{Experimental Results}

For our experimentation we generated 30 synthetic images, 10 of which are ideal, 10 have a Gaussian smoothing filter and 10 have a random weighted filtering applied. Also three real images have been manually labeled to compare our results against. Fig. 8 illustrates a manually labeled and automatically identified real image. The retinal cones have been identified with a black or white ' + ' symbol to allow for contrast in the image.
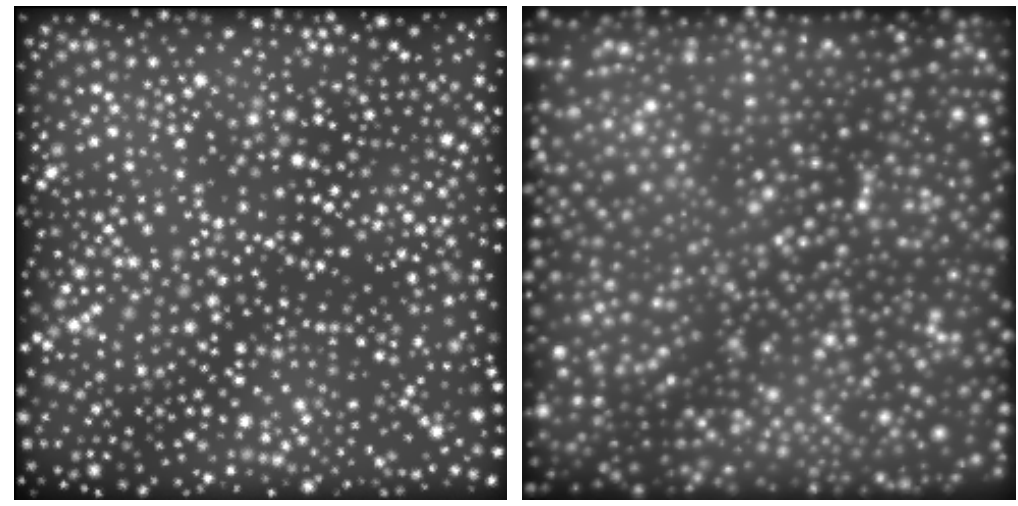

Fig. 7. Two example types of degradation applied to the synthetic images
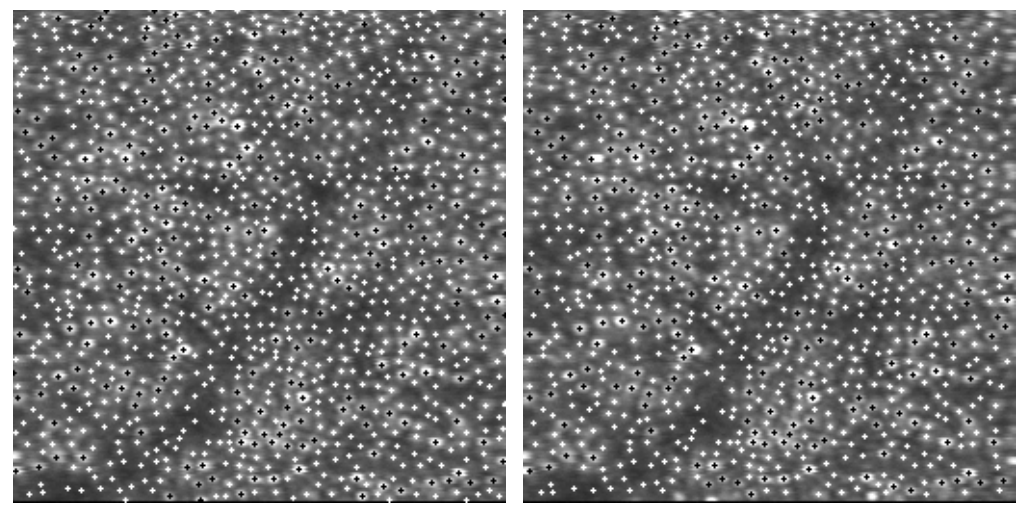

Fig. 8. A manually labelled and an automatically identified real image

Table 1 illustrates the results of our algorithms applied to the synthetic data generated earlier. These results have been separated into three sets, one for each of the synthetic data sets (ideal, Gaussian smoothed and random pixel shifted images). 
Table 1. Results of our algorithm against the different types of synthetic data

\begin{tabular}{|l||c|c|c|c|c|c|}
\hline \multicolumn{1}{|c||}{ Manual } & \multicolumn{2}{c|}{ Ideal } & \multicolumn{2}{c|}{ Gaussian Smoothed } & \multicolumn{2}{c|}{ Randomly Shifted } \\
\hline \hline Algorithm & $\begin{array}{c}\text { None Cone } \\
\text { Detected }\end{array}$ & $\begin{array}{c}\text { Cone } \\
\text { Detected }\end{array}$ & $\begin{array}{c}\text { No Cone } \\
\text { Detected }\end{array}$ & $\begin{array}{c}\text { Cone } \\
\text { Detected }\end{array}$ & $\begin{array}{c}\text { No Cone } \\
\text { Detected }\end{array}$ & Detected \\
\hline \hline Cone Detected & 8425 & 1 & 8664 & 135 & 8201 & 882 \\
\hline No Cone Detected & 390 & ---- & 422 & ---- & 466 & $\ldots--$ \\
\hline
\end{tabular}

This gives an average accuracy of $96.2 \%$ for correct cone identificaton. A relatively low percentage of $3.9 \%$ of the total number of photoreceptor cones were missed in our synthetic data. Also $4.8 \%$ of the total cones identified were false positives.

Table 2 illustrates the combined results of our algorithms when applied to the three real images (which have been manaually analysed).

Table 2. Results of our algorithm against real image data

\begin{tabular}{|l||c|c|}
\hline \multicolumn{1}{|c|}{ Manual } & $\begin{array}{c}\text { Cone } \\
\text { Detected }\end{array}$ & $\begin{array}{c}\text { No Cone } \\
\text { Detected }\end{array}$ \\
\hline \hline Cone Detected & 2364 & 145 \\
\hline No Cone Detected & 172 & ---- \\
\hline
\end{tabular}

When this is applied to our real image data we correctly identified $94.2 \%$ of photoreceptor cones and missed 5.7\%. Of the total cones identified $6.8 \%$ were false positives. These results clearly illustrate a good foundation for automatic retinal cone identification.

\section{Conclusions}

We have illustrated a process of automated photoreceptor cone identification using modelling and normalized cross-correlation. We have also presented a process for synthetic image data generation imitating the images obtained from a modified CSLO such as a HRT. This process has produced an accuracy of $95.2 \%$ on synthetic data and $93.2 \%$ on real image data.

Further work in this area would include modification of the modelling process in an attempt to increase the number of true positives found. Also the expansion of postprocessing algorithms to reduce the number of false positives would be required. The automated identification and masking of retinal blood vessels can also be implemented. With the identification of retinal blood vessels, the ability to automatically align these images on top of a high-resolution Fundus image should be possible. This provides a visual tool to identify how far away from the fovea the image has been captured. Further development of synthetic data may include the random placement of retinal blood vessels and the increased implementation of noise to simulate poor images. 


\section{References}

1. Miller, D., http: / /research.opt.indiana.edu/Labs / Adaptiveoptics/default.html

2. Li, K.Y., Roorda, A.: Automated Identification of Cone Photoreceptors in Adaptive Optics Retinal Images. J. Opt. Soc. Am. A. 24, 1358-1363 (2007)

3. Roorda, A., Zhang, Y., Duncan, J.L.: High-Resolution In Vivo Imaging of the RPE Mosaic in eyes with Retinal Disease. Invest Ophthalmos. Vis. Sci. 48, 2297-2303 (2007)

4. Roorda, A., Romero-Borja, F., Donnely, W., Queener, H., Hebert, T., Campell, M.: Adaptive Optics Scanning Laser Ophthalmoscopy. Opt. Express. 10, 405-412 (2002)

5. Roorda, A., Metha, A.B., Lennie, P., Williams, D.R.: Packing Arrangement of the Three Cone Classes in Primate Retina. Vision Res. 41, 1291-1306 (2001)

6. Wojtas, D.H., Wu, B., Ahnelt, P.K., Bones, P.J., Millane, R.P.: Automate Analysis of Differential Interference Contrast Microsopy Images of the Foveal Cone Mosaic. J. Opt. Soc. Am. A. 25, 1181-1189 (2008)

7. Curcio, C.A., Sloan, K.R., Kalina, R.E., Hendrickson, A.E.: Human Photoreceptor Topography. JCN 292, 497-523 (1990)

8. Nicholson, W.V., Glaeser, R.M.: Review: Automatic Particle Detection in Electron Microscopy. J. Struct. Biol. 133, 90-101 (2001)

9. Paques, M., Simonutti, M., Roux, M., Bellman, C., Lacombe, F., Grieve, K., Glanc, M., LeMer, Y., Sahel, J.: High-Resolution Imaging of Retinal Cells in the Living Eye 21, S18-S20 (2007)

10. Heidelberg Engineering, http: / /www . heidelbergengineering . com/ 